\title{
How to Implement a Small Blood Bank in Low and Middle-Income Countries Work in Progress?
}

\author{
Pierre Zachee ${ }^{*}$, Philippe Vandekerckhove \\ Red Cross International Blood Services, Belgium
}

\begin{abstract}
Compared to High-Income countries (HIC), a shortfall in the provision of blood remains a multifaceted problem in Low and Middle-Income Countries (LMIC) with a direct negative effect on clinical care. The reasons are multifactorial: not only lack of knowledge, skills, and resources, but also huge differences in environment climate, endemic transfusion transmittable infections, clinical set-up, availability of clean water, electricity. It is therefore obvious that simple translation of guidelines, standards, experiences, and the total organization from HIC to LMIC is not the best way to proceed. Adapted, but not less adequate methods for blood transfusion training, organization, and accreditation are required. The Global Advisory Panel (GAP) already formulated an adapted specific answer in terms of training and accreditation. But this is not enough. Academic centres, the GAP, countries, non-governmental organizations, and others need to test current and innovative diagnostic, production, and storage methods in a joint venture with the industry. Also, medical decisions focused on transfusion must be tested before implementation in facilities allowing pre-qualification of tests and devices. The entire transfusion chain needs to be simulated in a competence and training centre, focusing on the region where it will be applied. One of the renowned tropical institutes, currently fulfilling all these requirements could be the ideal place for such a competence centre. This review highlights this and suggests possible ways and solutions.
\end{abstract}

Keywords: Transfusion; Low- and middle-income countries; Global advisory panel; Blood donation

\section{INTRODUCTION}

Safe blood supply and the knowledge, skill, and resources to achieve this are essential for medical services. Many problems arise in the development of transfusion services in low- or medium-income countries (LMICs). Unfortunately, in many countries, providing safe blood is made much more difficult by a lack of blood donors and the high frequency of transfusiontransmissible infections (TTI). Urgent life-saving transfusions are frequent. Such as paediatric anaemia meanly with malaria, sickle cell anaemia, blood loss during pregnancy, delivery, trauma, and surgical interventions. A rather new category constitutes nontraumatic bleedings, with the largest single diagnosis being gastrointestinal bleeding. Transfusion indications are completely different in LMICs in comparison to high-income countries (HICs). With more planned transfusions, and a to support other Surgical and medical interventions: transplantations, cancer, dialysis, etc. in HICs. Other differences between HICs and LIMCs is the transfusion index, differences in blood fractions used, and the blood product use per population. The transfusion index is defined as the number of products per transfused patient. This is much lower for LMICS compared with the United States (1.65 and 2.72, respectively). Similarly, the use of the different blood products varied between LIMCs and high-income countries such as the United States, with $58.5 \%, 31.7 \%, 7.2 \%$, and $2.6 \%$ versus $66 \%, 0.1 \%, 19 \%$, and $14 \%$ for RBCs, WB, FFP, and PLTs, respectively [1]. Possible explanations for these differences, especially the high percentage of WB use in LMICs, could be associated with limited clinical awareness and the high cost of component separation, as well as the local blood demand for WB [2]. Compared with highincome countries, blood product use per population in LMICs is also incredibly low. The WHO estimates of 32.0 RBC units (range, 7-49) per 1000 population and 3.41 RBC units (range,

*Correspondence to: Pierre Zachee, Professor, Red Cross International Blood Services, Belgium, Tel: +32495202291; E-mail: pierre.zachee@vrijwilliger.rodekruis.be

Received: May 03, 2020; Accepted: May 21, 2020; Published: May 27, 2020

Citation: Zachee P, Vandekerckhove P (2020) How to Implement a Small Blood Bank in Low and Middle-Income Countries Work in Progress. Trop Med Surg. 8:227. doi: 10.35248/2329-9088.8.1.227

Copyright: (C) 2020 Zachee P, et al. This is an open-access article distributed under the terms of the Creative Commons Attribution License, which permits unrestricted use, distribution, and reproduction in any medium, provided the original author and source are credited. 
0.32-10) per 1000 population in high-income and low-income countries, respectively [3]. The low transfusion rate in IMICs is linked to a lower donation rate, which in turn is to a great extent a result of inadequate funding. WHO data estimate donation rates of only 4.6 donations per 1000 population per year for low-income countries. However, to align with the World Health Assembly resolution WHA63.12 on availability, safety, and quality of blood products, LMICs nations need to improve blood availability to meet the increasing demand for transfusion [4]. National blood transfusion services (NBTSs) are already established in most LMICs since the early part of this century in line with the World Health Assembly resolution WHA28.72 on the use and supply of human blood and blood products. While these services have improved the situation, shortages remain.

Cultural, climatological, and epidemiological differences are so pronounced that it is obvious that transplantation of blood banking habits and standards from HIC to LIMC is not a good idea for many reasons.

We have identified five pillars that should guarantee the establishment of small blood banks in LMIC: Innovation, prequalification analysis, guided operational implementation, performance qualification, and education.

The transfusion act am a chain with many links each interacting with each other. If one link fails, it has a repercussion on the whole chain. The transfusion chain can be divided into consecutive steps, as depicted in Figure 1.

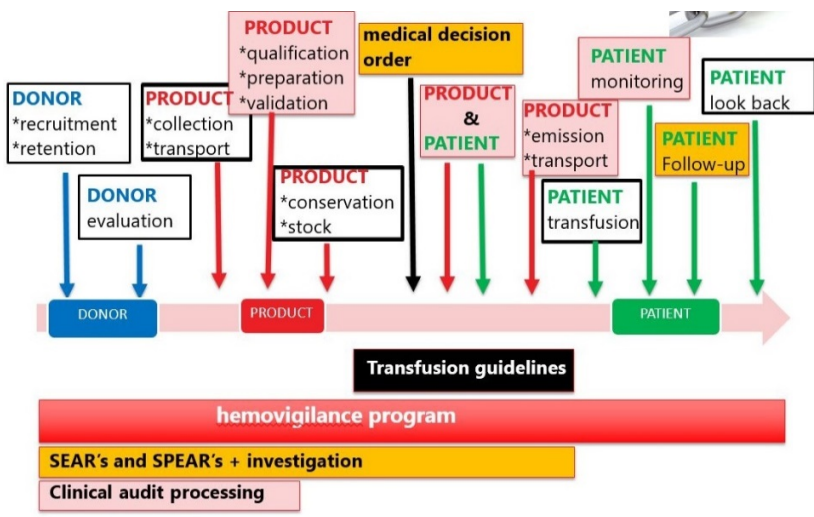

Figure 1: The transfusion chain.

In the first part of this article, with the experience, we have gained in South Africa, Laos, and Republique Démocratique du Congo, but also based on knowledge gained in the Tropical Institute of Antwerp (ITM) and extensive literature search, we will focus on Innovation, pre-qualification analysis, guided operational implementation and performance qualification. We will examine each step. The challenges which are being encountered in LMICs, predominantly focusing on sub-Sahara Africa (SSA). We will also provide adapted and cost-effective answers on each link of the transfusion chain adapted to be applied in the former setting. Each measure that is introduced must be mirrored on the cost per QALY or DALY and the gross domestic product per capita (GDP) of a certain country. This exercise can only be made for a specific country or situation and transcends the broad horizon that we have chosen for this review. In the second part, we will shortly describe the education tools which could be provided by the global advisory panel on corporate governance and risk management of blood services in the Red Cross and Red Crescent Societies (GAP).

Innovation, pre-qualification analysis, guided operational implementation and performance qualification of the Transfusion Chain

DONOR recruitment and retention: Blood donor selection remains the first level of defines against transfusion-transmitted infections (TTIs); the deferral of high-risk prospective donors is a primary strategy to reduce risk. The favoured source of blood collection is voluntary and no remunerated blood donation (VNRBD) because such donors have been found to have a lower risk for TTIs, at least in developed countries. VNRBD was first defined in 1975 at the World Health Assembly (WHA). The Melbourne Declaration on '100\% Voluntary non-remunerated Donation of Blood and Blood Components' founded on the policies articulated in World Health Assembly resolution WHA28.72 and WHA58.13 urges the Member States to promote the development of national blood services based on voluntary non-remunerated blood donation and call for action to all governments to achieve $100 \%$ voluntary non-remunerated donations by 2020 as the cornerstone of their blood policies.

\section{Ethical code}

Replacement donation (RD); defined as a donation from family members, relatives, or friends of the patient, is the major alternative to VNRBD in Africa is the major alternative to VNRBD and the primary source of blood collection in much of the African countries. Estimated to contribute $75 \%$ to $80 \%$ [3].

However, the distinction between replacement and VNRD donors is not always clear-cut. We and others identified different domains of comparison: supply, quality, cost, quality and safety [5]. The supply of VNRBD blood are typically recruited through a dedicated and centralized system, for example, blood centres independent of the hospital. Centralized blood centres require a developed infrastructure to be sustainable for storage, refrigeration, transportation, communication, and QA. All these items are often poorly developed in setting with poor resources. Deficiencies in transport and storage have particularly adverse effects in Africa where blood is prescribed for medical emergencies; the ability to tolerate delays, incumbent to procuring blood from centralized blood centres outside the hospital, is poor [6]. VNRBD requires strategized recruitment, marketing, and collection to secure and, consequently, retain enough donors to answer the demand and is logistically complex. Replacement donation is logistically easier than volunteer donation as it does not require the infrastructure or recruitment mechanisms associated with VNRBD. But this is to the detriment of households were the burden, cost, and responsibility for recruiting donors shifts to the patient's family and friends. And have so, in addition to caring for a sick family member, to bear an extra emotional and economic burden on their shoulders. The success of RD is the result of the strong cultural ties and extensive family support that is visible in many parts of Africa. 
Frequent shortcomings of RD include also inadequate testing of units for TTI, lack of QA, and poor record-keeping because of the collection in the hospital setting where transfusion oversight is often poor [7]. The latter may partly account for the enormous variability in TTI data on RD. The differences in recruiting donors, quality assurance processes, and maintaining distribution networks are reflected in the cost. A unit of blood collected through a centralized system is 2 to 3 times more expensive than that collected through RD. The hospital-based replacement donor system existed for a long time and has, therefore, demonstrated that it is sustainable. In contrast, with very few exceptions, the centralized systems are not viable without external funding [2].

\section{Concerning safety}

$\mathrm{RD}$ was long been regarded as a higher risk based upon the assumption that friends or relatives are more likely to deny or ignore risk factors that invite further inquiry, removing the protection afforded by risk-screening questionnaires in favour of perceived coercion to donate. Family members may also be at higher risk than VNRBD for some TTIs because of ethnicity, for example, the hepatitis B virus (HBV). Furthermore, were burdened with the responsibility to procure donors urgently, family members may pay donors for their services, thereby aggravating the transfusion risk.

But is one RD who gives blood for altruistic reasons equal to another RD who will be paid? VNRD receives some form of compensation such as refreshments, transport costs, and small gifts. This is the difficult balance between unacceptable rewarding and allowable recompense [8]. Is a one-time RD equal to a repeat RD? About these recent studies, however, need to be considered. They pointed to a lack of difference between the prevalence of anti-HIV and hepatitis B surface antigen in the first-time VNRBD vs RD in Africa and Latin America $[9,10]$. First-time donors are considered high risk for HIV and other TTIs; these donors are usually young with a concomitant higher prevalence of sexually transmitted TTIs, have uncertain or untested motivation to donate; many first-time donors use the donation to have access to free HIV testing. Also, there is no published evidence comparing the safety of replacement repeat donors and voluntary repeat donors, so it is not known whether replacement donors who donate regularly are less safe than repeat voluntary donors. This has prompted many to rethink the validity of exclusively advocate the VNRBD and to closely reexamine the risk attached to any given subset of the donor. Country-specific TTI prevalence data are needed on first-time vs repeat donor status when making comparisons between VNRBD and RD.

Another especially important safety measure is the need for a disconnection between blood product providers and blood product users. Without this barrier, there is a chance that users are under high pressure to accept sometimes less secure donations. Also, to prevent commercialization by malicious people, which would inevitably lead to unsafe practices, that I could observe. Voluntary donors are recruited through a centralized system, for example, a national blood transfusion service, that remains independent from the hospital and is under legal supervision and with the financial support of a central competent authority.

Beyond the debate of VNRBD and repeated RD, insufficient recruitment of blood donors remains an enduring problem in much of Africa. The shortfall in recruitment is a complex problem rooted in culture, education, and marketing. The shortfall in the provision of blood remains a multifaceted problem in Africa with a direct adverse effect on clinical care.

Given all these constraints novel strategies need to be adopted to facilitate VNRBD. Strengthening the existing replacementbased model through on-site recruitment of voluntary donors, investment in independent hospital transfusion services with testing, QA, and data collection may be preferable and improve the transfusion shortfall. Further consideration of RD vs. VNRBD ought to be driven by data on TTI prevalence and/or incidence in the 2 types of donors in the local setting. Many replacement donors share similar altruism to VNRBD and can be used effectively while still maintaining transfusion safety. Strategies of donor retainment to convert first-time RD donors into regular donors could be successful [11-13].

Donor profiling and risk assessment are based on the availability of robust epidemiological data, revisiting the fundamental need for situational analysis. This includes demographic and behavioural risk factors for sexually and parenterally acquired infections, for example, HCV, HIV, and syphilis. Knowledge of climatological, geographic risks for infections, as e.g. malaria and arboviruses are important. To some extent, collection practice and donor deferral can be adapted accordingly, particularly if a risk is localized, e.g. West Nile, Dengue, Zika, chikungunya (CHIKV). It becomes more difficult to deal with the impact on the donor pool if the infectious agent is hyperendemic to the area as in the case of malaria.

Organizations such as the WHO, the GAP, and the IRFC are struggling with the difficulty of proposing new strategies in this regard. This is since the situation in the field can be quite different from region to region. What is good for one can turn out completely differently for the other. There is also the fear that solutions provided from a central organization will not be accepted by the local population and could even be perceived as neo-colonial. What the WHO, the GAP or the IRFC can propose is a method that could be applied region by region, and in which the local population is not only part of the decision pathway, but also the owner of it. This method is based on four steps.

It starts with a situation or operational analysis by a model to seek explanations as it was done for tuberculosis [14] or to consider supply and demand constraints. The principal role of this first step is to identify bottlenecks.

The second step will Identify the key elements of the problem, the long-term outcome, and the policy options (action model). The objective is to assess how the local population sees the problem. The policy is supposed to solve the problem (e.g. diminish blood shortage, improve clinical care, etc.], and to define the policy options to reach these objectives. The process starts with the ideas presented by a wide range of local participants with different backgrounds. Afterward, ideas will be 
part of a discussion. Ideas will be reorganized and structured. Care must be taken to unpack and specify fuzzy definitions and vague statements. Facilitators will probe for elements on which a consensus exists and stimulate discussion on items where diverging ideas persist. Finally, to end up with a shared understanding of how the participants define the problem, the desired long-term outcome, and the policy options.

The third step is the pathway of change. Participants will discuss in small groups how they think the policy option(s) they propose will reach the long-term goal ['the intervention'] for the beneficiaries, the target groups, the expected effects, or results, and the potential negative effects. Groups will then present their action model and discuss and compare them with the other groups' results. As a result, the views of the different groups will be incorporated, issues where opinions diverge, and rival program theories will be identified. In the second part of this step, the groups will identify the following elements of the pathway: the mechanisms of change, the essential conditions for success (or the contextual constraints).

In the fourth and final step pathways are reviewed and rearranged if needed in the form of the core pattern of a realistic evaluation scheme: Problem, policy, actors, objectives, context conditions, expected results, and mechanisms of change. Coherence between problem identification-actors-policy objectives-policy components-context elements-expected results (positive and negative)-required conditions-mechanisms will be reviewed. (Knowledge) gaps will be identified, and solutions proposed and discussed. As a result: a structured pathway that summarises the program of the participants will be put on paper [15].

This method can also be used in the implementation of improvement actions in the later links of the transfusion chain. An example of this is given by Owusu-Ofori, describing the benefits of pre-donation screening now in routine use in several SSA countries [16].

Already programs such as school/university programs, corporate programs, Club 25 (through which secondary school donors pledge to donate $25 \mathrm{U}$ during their lifetime), and donor clubs are implemented and working [17].

In conclusion studies consistently demonstrate that the safest group of blood donors are those that donate blood regularly ["regular donors"]. These donors are well informed about the importance of leading a healthy lifestyle with low risk behaviours, to improve blood safety. Retaining regular donors also ensures the sustainability of the blood supply costeffectively. Strategies, adapted to regional differences, need to be implemented to target family replacement donors that have tested negative for transfusion transmissible infection (TTI) to convert them to regular voluntary blood donors. GAP provides an educational tool to reach these goals: The modules on voluntary none remunerated blood donation is in the section of the website entitled "PRIMARY PREVENTION MODULES" on the IFRC site.

\section{DONOR EVALUATION}

An adequate donor evaluation remains the cornerstone to provide efficient and safe blood products. This evaluation must be done in a standardized way and following National donor selection guidelines based on National/local epidemiological data on infectious diseases, nutritional and health status of the general population, and local customs. Also, health and safety outcomes of donors and recipients. International best practices need to be considered as e.g. the WHO/IFRC Pre-donation counselling and donor selection guidelines.

These guidelines should be reviewed regularly. These selection guidelines are important to guarantee the safety of the donor to donate blood, to reduce the risk of (TTI) or other adverse effects to blood recipients, to protect blood service staff and to build trust in the safety of the blood service

The evaluation of the donor is often not done in a gentle, comfortable, and confidential way, because of a lack of an adapted location (schools, marketplaces, churches) and under family or community psychological pressure. For this reason, adapted pre-donation questionnaires are needed in which sensitive questions on risk behaviour, which could lead to TTI loaded products are pooled together with less intrusive questions like a recent flue or fever. Questions on connectivity risk behaviour rather than their risk behaviour are as proven by the connectivity theory must more relevant. You could ask " do you think, your husband or your wife is faithful". Rather than " did you had several sexual partners last six months". Strong associations between the national prevalence of various STIs suggests sexual network connectivity is a common underpinning risk factor [18].

The relevance of each question to exclude a potential donor must be prospectively and continuously be evaluated. The ideal method is to determine the excluding power (EP) of a specific question [19].

Two potential useful exclusion methods can be introduced. The first during the donation act itself where every donor with a link to the donation deposited a document in a closed box on which he ticked whether his blood may or may not be used. A second after the blood donation where the donor informs by telephone or in another way that his blood may not be used. For example, because he became ill shortly after the donation. Both deferral methods are useful to relieve peer pressure on an individual donor.

The donor pool in Africa is not only affected by TTI's but also by malnutrition, infectious disease, and anaemia. All of which directly or indirectly affect donor eligibility.

To avoid donor adverse reaction like fainting and poor product quality due to low haemoglobin content a simple and cheap predonation haemoglobin determination is mandatory. Although the most widely used method at virtually no cost is the copper sulphate method with concentration adjusted to reflect a cut-off variable according to countries. New methods could be introduced on reliability, precision, accuracy, price, minimal equipment needed, test difficulty and staff training level and fit for purpose criteria. Relying on these criteria the Haemoglobin 
colour scale or the Lovibond comparator methods are put forward. Price respectively: 0.12 and $0.15 \$$ per test.

New non-invasive haemoglobin measurement methods need to be evaluated for specific populations.

- Masimo Pronto.

- Biosens Touch.

- Haemospect.

- Orsense.

In conclusion: Donor evaluation needs to be adapted to regional differences in setting and resources. Specific questionnaires need to be developed and the excluding power of each question needs to be assessed. Innovations could be considered.

\section{PRODUCT COLLECTION AND TRANSPORT}

The collection must take place in a clean, spacious, airy, well-lit, and quiet environment, with appropriate equipment: needles and disposable equipment.

Hygiene, health, and the protection of personnel must be the subject of a procedure drawn up by those responsible. This is technical well explained in several officials WHO documents.

As one could imagine this is far from ideal in LMIC. There is a tremendous need for education.

Also, prequalification for in vitro diagnostics and medical devices for blood collection in LMIC is needed. Which devices are optimal for use in tropical areas? Which diagnostic tests are appropriate in such circumstances with the focus on blood donation? These are essential and appropriate questions.

The process of prequalification of in vitro diagnostics and medical devices is recently set up by the WHO. The focus, however, lays more on patients than on blood donors. The World Health Organization (WHO) Prequalification of In Vitro Diagnostics (IVDs) is coordinated through the Department of Essential Medicines and Health Products (EMP). Focus is placed on in-vitro diagnostics for priority diseases and their suitability for use in resource-limited settings (Figure 2).

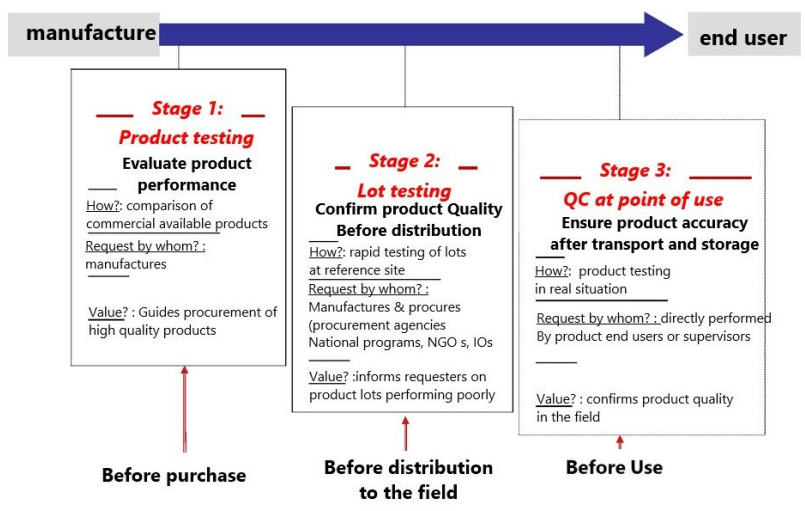

Figure 2: Strategy to prequalification.

This WHO document provides manufacturers of WHO prequalified in vitro diagnostic medical devices (IVDs) with information on when and how they must report to WHO.
In conclusion besides the need for education, which will be covered by the GAP initiative, there is a need for one or several worldwide centralized institutions, with extensive knowledge of tropical and subtropical environments. They also need to have experience with the limitations tied to socio-economically disadvantaged regions. They need to be able to create joint ventures with the industry, academic centres, the GAP, countries, non-governmental organizations, and others on current and innovative diagnostic, production, and storage methods. Medical devices focused on transfusion must be tested in advance. The competence centres could play a role in the prequalification of tests and devices. Ideally, the entire transfusion chain should be simulated in a competence and training centre, focusing on the region where it will be applied. To support the existing regulatory institutions in LMIC whose function is to approve or reject instruments, materials, and tests applied to health care including transfusion.

\section{PRODUCT QUALIFICATION, PROCESSING AND RELEASE}

\section{Qualification}

Screening: The risk of transmitting pathogens by a blood transfusion in LMICs is very high, not only because many bloodborne pathogens are endemic and region-specific, e.g. Malaria, but also due to insufficient facilities, funding, governmental awareness, and centralized safety and educational programs.

High-performance serologic testing and nucleic acid testing (NAT) are the cornerstones of TTI screening. The main advantages of NAT screening are the prevention of new incident cases during the window period infections, the identification of occult hepatitis B carrier status, which can potentially be infectious, and the fast implementation in the case of Emergency Infectious Diseases (EID), as we encounter now with Covid-19 [20]. Most LIC can neither afford the use of NAT nor have the trained personnel, infrastructure [21].

In 2016 only 6 of 46 surveyed African countries reported 100\% screening coverage for the major TTIs (HIV, HBV, HCV and Syphilis); much of the testing was also performed using rapid diagnostic tests (RDTs) [3].

Given the low cost, no stable power supply and relative ease of use, RDTs are widely used for donor screening in SSA, despite having been shown repeatedly to have lower sensitivities and specificities than enzyme immunosorbent assay (ELISA) and (NAT). RDT is intended for patient care and not for donor screening. In patients, a second and sometimes a third test is needed to confirm the diagnosis in both RDT negative and positive cases. In Donor evaluation, the most sensitive test is needed to exclude a donation. This is a combined antigen and antibody 4 de or 5 de generation ELISA test. Remember both the confirming and the excluding power of an argument increase by increasing the sensitivity and the specificity, but the former is much more affected by specificity, the latter by sensitivity [22,23]. Another problem is the prozone effect in RDT on heavily contaminated samples. 
To ensure blood safety, the recruitment and retention of voluntary, non-remunerated repeat donors should be a major commitment for low resource countries in which NAT implementation is costly and not feasible [24].

Nevertheless, the development of NAT tests, which are adapted for LMIC need to be further investigated. One example is the loop-mediated isothermal amplification. Another option is to screen by mini-pool NAT testing.

Pathogen prevention, inactivation, and removal: Another approach that can be considered is pathogen prevention, inactivation, or the removal of pathogens. Various technologies are possible: collection techniques, temperature and time, filtration, pathogen inactivation, treatment of the recipient during transfusion.

Bacterial contamination is one hundred times higher in SSA in comparison to Europe. A recent study in DRC (Democratic Republic Congo) reveals bacterial contamination of $0.3 \%$ in whole blood and $1.4 \%$ in Packed cells. Simple collection measures like combined skin disinfection and diversion of the first $\mathrm{ml}$ of collected blood can reduce bacterial contamination by $77 \%$.

-Due to the bactericidal and bacteriostatic properties of blood components like white blood cells (WBCs) and complement proteins, the survival and growth conditions for most bacteria are poor in fresh whole blood. In the past, it was advised to keep the whole blood collection bags for at least four hours at room temperature $\left(18-20^{\circ} \mathrm{C}\right)$ before further separation into components to take advantage of this effect. As scientific data are rather old, this practice needs to be reassessed [25]. It is believed in most health facilities, based on tradition, that $T$. pallidum spirochetes are fragile and cannot withstand blood bank temperature when subjected to it for many hours. This is mainly based on experimental studies in rats. However, it is fully unclear whether infectivity in rat testes can be paralleled with infectivity in the human system. Also, the disease progression in animals versus humans may be quite different. Therefore, it is advised that $T$. pallidum should be screened before allogeneic transfusion [26].

It is further clearly demonstrated that other cold-sensitive microorganisms, such as Borrelia burgdorferi, Ehrlichia Bartonella, Trypanosoma cruzi, Trypanosoma Babesia could survive quite a long time in refrigerated blood [27-30].

Leukodepletion filters of different types are designed to be used at the time of collection or bedside. Leukodepletion filters have been widely used to reduce transmission of organisms such as cytomegalovirus, which exists intracellularly within WBCs. Leukodepletion has also been used to prevent transmission of the intracellular human T-lymphotropic virus-I and II. However, not always successfully, when evaluated with molecular techniques [31]. It is the same accounts for the rickettsial species Orientia tsutsugamushi [32].

Although Trypanosoma cruzi is not an intracellular organism, leukodepletion filters have been suggested as a means of reducing this organism in infected blood, due to their adherence properties to the leukoreduction filter. Leishmania spends part of its life cycle as an intracellular parasite in monocytes and macrophages and part as an extracellular organism in the insect vector. This parasite survives in both forms in stored blood products. Leukoreduction filters have been shown to remove both forms of the organism by removing the WBCs containing the organism and by direct adherence of extra-cellular organisms to filter fibres.

The recent increase of Ana plasma phagocyto philum may represent an EID blood safety issue. In the absence of reliable testing leukoreduction by filtration may offer some protection [33].

Concerning bacterial contamination of blood products, leukoreduction filters have also been suggested as a means of decreasing the incidence of bacterial overgrowth [34].

There was an unexplained decrease in the incidence of transfusion-transmitted malaria in recent years. The decrease in incidence has paralleled the increasing use of leukoreduction filters. Leukoreduction filters are not able to remove red blood cells (RBCs) infected with parasites from blood products, because these filters are designed to allow RBCs to pass while trapping WBCs and platelets (PLTs). There are, however, reasons to suspect that changes that occur in malaria-infected RBCs may lead to adherence of infected RBCs within these filters. There were at least three mechanisms of adherence of malaria-infected RBCs demonstrated within the filters. The first was the adherence of infected RBCs to other RBCs; the second was direct adherence of infected RBCs to filter fibres; and the third involved adherence of malaria-infected RBCs to platelets, which were adherent to filter fibres. Filtration also resulted in preferential removal of phosphatidylserine (PS)-expressing cells as seen by the reduction of annexin $\mathrm{V}$ binding after filtration [35]. Leukodepletion filters are widely available and could be implemented immediately in at-risk areas. Further systematic efficacy analysis in a stand-alone situation or combination with other techniques is mandatory.

While potentially beneficial for some patients, leukoreduction by filtration in SSA would likely adversely affect the already limited blood supply. The reason is that the prevalence of sickle cell trait (i.e. heterozygosity for Haemoglobin AS) is high in SSA: $13 \%$ in Ugandan new-borns and $22 \%$ in a study of Nigerian adults. Sickel Cell trait carriers are asymptomatic and therefore able to give blood, but filtration blocking is much higher with AS blood than AA blood [36].

It is believed that pathogen inactivation (PI) could be an alternative strategy for biological testing for TTIs. PI refers to the global inactivation of infectious agents in blood products using various technologies. Various technologies are possible including mechanical disruption (used in plasma processing) by heat treatment or nanofiltration or photochemical handling of platelets and/or plasma with solvent detergent photoactive methylene blue or psoralen/riboflavin inactivation of DNA and RNA. These methods differentially affect a variety of viruses, bacteria, and protozoa (including malaria) incapable of replication and produce infection.

Different PIs exist and are currently used: Mirasol®, Intercept ${ }^{\circledR}$, and Thera flex ${ }^{\circledR}$. These procedures use a chemical and 
ultraviolet or visible light. Most PI technologies are approved for platelets and plasma. In contrast, WB, and red blood cells (RBCs) constitute the major prescribed products in SSA. Examples of PI use in SSA are few, having largely been confined to emergency use (e.g., Chikungunya outbreak in Reunion 2005) [37].

A clinical trial in Ghana demonstrated a significantly reduced incidence of transfusion-transmitted malaria (TTM) in those who received Mirasol (Terumo BCT) pathogen-inactivated WB [4\%] as compared to untreated standard WB transfusions [22\%] [38].

Experimental data indicate that the Mirasol System could be effective at preventing the transfusion of Trypanosoma Cruzi [39]. Also in vitro studies confirm the effect of Mirasol on the Ebola virus [40].

leukoreduction and Riboflavin and UV light treatment used in conjunction appear to eliminate a significant amount of viable T. cruzi in whole blood. These combined strategies could complement other blood bank measures already implemented to prevent the transmission of T. cruzi via blood transfusion [41]. PI could benefit regional blood safety; however, although there are many obstacles to broader implementation include lack of skilled personnel, costs, infrastructure, and the technical complexity [42]. PI does, however, holds promise and will hopefully gain momentum in the next future. However, additional safety issues need to be further solved [43].

There are no screening tools for malaria that are affordable, practical, and suitably sensitive for use by blood banks in SSA. The prevalence of malaria in blood donors is variable but in some parts of West Africa could reach 55\%. Implementation of any policy that advocates deferral of all such donors will have a significant negative impact on the availability of blood products and will so undoubtedly increase mortality particularly among children and pregnant women [44]. The administration of subsidized and low-priced anti-malarial prophylaxis with every transfusion appears a practical alternative strategy to lessen Transfusion transmitted malaria (TTM) in SSA. One should, however, bear in mind that complete prevention of TTM may not be attainable, so malaria must always be considered in any patient with a febrile illness post-transfusion.

This post-transfusion treatment strategy could also be used for other TTI.

In conclusion all these removal techniques need to be reassessed one the one, combined and prequalified.

Other tests finally, biological testing is not confined to infectious screening and extends to immuno-hematological testing, as $\mathrm{ABO}$ and compatibility testing.

In the WHO survey, all countries in the region reported $\mathrm{ABO}$ cell grouping of donors, yet only $71.8 \%$ reported indirect serum grouping (Simonin method) [45]. Various subgroups of A and $\mathrm{AB}$ that are known to be prevalent in the black population will not be detected using this method and can precipitate acute haemolytic transfusion reactions.
In the population of African descent, a high frequency of partial Rhesus antigens is present. A partial antigen is defined by the lack of some immunogenic epitopes. A patient with partial D antigen can develop antibodies against the missing epitopes when exposed to the conventional D antigen.

Additional screening is needed for donors for sickle cell disease and major enzymopathies, for example, G6PD deficiency, all of which have clinical implications for prospective recipients.

Improvement in immunohematology training, QA, and availability of reagents certainly warrants attention [46].

Product Processing: Rational blood use also includes efficient blood use. Component therapy is a means to improve efficiency through differential fractionation of whole blood into derivative red cells, platelets, cryoprecipitate, and plasma. Whole blood needs to be transfused to iso 'ABO' type-matched recipient given the large antibody-containing plasma fraction. In contrast, a group ' $O$ ' red cell component can be transfused safely into any recipient, but a group ' $O$ ' unit of whole blood is restricted to group ' $\mathrm{O}$ ' recipients unless established to be of low antibody titter.

In sub-Saharan Africa whole blood transfusion is still the most used form of transfusion [1]. The large volume of whole blood places recipients at risk for transfusion-associated circulatory overload, as in young children. Accordingly, one important processing consideration is the availability of partial whole blood or red cell units. This is a major reason for the preparation of red cell units in some African blood centres, where whole blood is collected into a multiple bag system, the plasma is removed, and two or three 'paediatric RBC packs' are prepared.

Processing measures that are routinely used to reduce transfusion complications are neither available nor affordable in most LMIC. These measures include pre-storage leukoreduction, gamma-irradiation, and avoidance of plasma-containing components from male donors (as a transfusion-related acute lung injury (TRALI) prevention strategy).

So, it is appropriate to introduce fractionation widely, starting with fractionation of whole blood into packed red cells and plasma. In a second stage into the other components. Fractionation allows not only to introduce the concept of the universal $\mathrm{O}$ negative donor but also to the rational use of different blood fractions. But without losing sight of the fact that fractionation and component strategy could lead to massive wasting of FFP. A possible solution for this is dry frozen plasma [47].

When fractionation is introduced, the concept of "secured quarantine frozen plasma" can be introduced. And its advantage that can be used now.

Similarly, blood centres may use different security measures for quarantine. Quarantine relies on donors coming back to make a new blood donation or to provide a sample for testing before the stored FFP can be cleared for release. This quarantine could be passively or actively organized. Passive quarantine relies on donors returning for the subsequent donation, such as calling the donors, to seek the donors' return. Active quarantine is 
where donors are contacted and asked to return as early as allowed in the quarantine period: maintaining such a program requires large human and other resources. When the window phase of a TTI has expired, and a new test for that TTI is negative, the plasma can be considered safe. This is certainly an additional safety measure for a first-time donor [48].

Others have argued that the complete conversion of a LIC's blood supply from whole blood to components is not always the best option: it is more expensive to prepare components, much of the blood in LIC is used to treat acute haemorrhage e.g. massive bleeding and in the post and peripartum bleeding. Even in HIC, several randomized studies are currently underway, to prove the benefit of fresh whole blood in these settings [49]. It is an area of blood transfusion where 'one size' does not fit all [50]. However, some access to components is important even in LMIC as they gradually introduce more sophisticated medical and surgical treatments. The optimal balance of whole blood vs. component preparation is likely best determined locally or regionally, taking into consideration regional indications for transfusion and available resources.

Product release: National analysis algorithms need to be defined to describe the required process of analysis and management of the results of each donation. These algorithms have to ensure the consistency of blood tests and interpretation of results by imposing the precise sequence of analysis (i.e, initial tests, control test, and any additional or confirmatory testing), the follow up on the use and destination of the blood component according to the results of the analysis. These algorithms should also describe the measures to be taken in donor management, namely donor exclusion, notification, counselling, and, where appropriate, re-entering analysis, again to guarantee consistency of implementation.

The same four steps method, as described in the chapter donor recruitment and retention, can be used for the implementation of these national algorithms.

In conclusion product qualification, product processing, and release is the most challenging step in the transfusion chain. Education, training, accreditation, innovation, research, and pre-qualification are central here. All the previously formulated partial conclusions are also relevant here.

\section{Product storage, stock}

The preceding steps in the transfusion chain are expensive and time-consuming, but of course extremely important to be able to deliver a safe product. It is therefore obvious that a balance must be found between production and consumption. And it would be a shame that there is not enough blood available, but even worse than too many blood products must be thrown away because the expiry date has been exceeded. Blood availability could be seasonal mostly depleted during school recesses and, in some areas, Ramadan. Recommendations should then be made to increase collection in the range of social events, particularly at radio stations [17].

Instead of having all blood groups in stock, one can limit oneself to an urgent stock consisting of universal donors. An ambulatory on-call tested donor pool, with various blood group types should, also, be available, which can be called up immediately. This is called the principle of walking donor transfusion bank. This method is used in military operations and mass casualty. But also, in LMICS. A specific risk assessment for TTI is therefore required for this specific form of donation [51].

The characteristics of a universal donor can differ regionally. Just think of the Rhesus or Duffy factor in Africa. The stock must then be limited to the blood group that covers the characteristics of $95 \%$ of the recipients.

The storage infrastructure must also be adapted to the needs: quantity of products to be stored, type of cooling capacity (vertical and horizontal), range $\left[4^{\circ} \mathrm{C}-\right.$ minus $60^{\circ} \mathrm{C}$ ), housing, energy supply, and local climatological factors. A prequalification of this infrastructure is therefore necessary.

In conclusion new forms of stock management and adapted infrastructure must be implemented.

\section{Patient: Medical decision to transfuse}

Despite the shortfall in blood availability, there continues to be inappropriate blood use and concomitant wastage. This exposes patients unnecessarily to the hazards of transfusion and, when coupled with deficient biological testing, this increases the already high risk of TTI.

Over-transfusion stems, in part, from lack of defined policy or clinical guidelines for appropriate use either at a national or local level. Lack of education and training among prescribing physicians contributes to liberal transfusion practice against illdefined thresholds.

The level of training among prescribers also varies greatly. In some cases, nurses act alone to prescribe transfusion [52]. Access to educational programs improves transfusion practices and helps to build a skilled workforce: The African Society for Blood Transfusion (AfSBT) has promoted education and training over the past 5 years, having established a pool of educators to provide training on the AfSBT standards, basics of blood safety, and to prepare transfusion services for accreditation.

A lack of diagnostic resources does not make it easier. The transfusion service is also frequently fragmented with little interaction between the blood center and the hospitals or prescribing physician and thereby precludes the monitoring of transfusion practice. Only a few hospitals have transfusion committees to regulate prescribing [53].

Strategies addressing the need for a new way of thinking adapted to the local situation is obvious. But also making new ways of clinical decision making in an area with restraint diagnostic and therapeutic resources. Methods to overcome this are "Vertical analysis of a transfusion problem", "A diagnostic panorama”, "the threshold to take a decision".

Vertical analysis (VA) is an analytical methodology applied to a health problem, developing a strategy tailored to the context [54]. We must also realize that blood transfusion is only part of a therapeutic algorithm. These treatment algorithms are the best cast in a previously defined workflow, where each component is 
described in detail, and applicable in each setting. For example, for anaemia in a young child with acute falciparum malaria, resuscitation with glucose, anticonvulsants, oxygen and antibiotics and artesunate will also be included in the treatment schedule and it will be determined when a transfusion is justified. A transfusion will thus get its proper place in a treatment schedule and it is prevented from unnecessary use [55].

Also, the implementation of policies, "bloodless medicine" or conservative transfusion practice are well described as a successful adjunct strategy to protecting transfusion inventories while still preserving sound clinical care. This incorporates a range of interventions that limit unnecessary blood use, many of which can be tailored to a resource-poor setting. These include the use of conservative transfusion thresholds; alternatives to blood transfusion such for example, iron and folate; and attention to haemostasis and surgical technique.

Whit a diagnostic panorama we want to develop our diagnostic landscape to include the following information about a specific clinical presentation: possible diagnoses, whether they are 'important', how we can confirm or exclude them, and which diagnoses have similar clinical presentations.

The final decision, for any given hypothesis in our 'diagnostic panorama', to transfuse or not will be determined by the minimum acceptable level of probability, or certainty This is the decision threshold. Whit the decision threshold we determine that we have reached a sufficient level of probability by summing up the different confirming and excluding powers from different clinical signs, tests, situations and determinants [56].

In conclusion in a setting with low resources, new paths need to be explored to arrive at a diagnosis and treatment. Here is explained how to draw a diagnostic panorama, to determine a threshold, and to follow situation-oriented comprehensive algorithms. So, we can steer our transfusion capacity in the right direction.

\section{Patient: Crossmatch donor/patient}

The determination of an ABO blood group comprises two tests, a globular test (called Bet Vincent) which makes it possible to search for the antigens carried by the red cells of the test subject using anti-A and anti-B antisera, and a serum test (called Simonin) which highlights the antibodies Anti-A and/or anti-B present in the serum using red blood cells of blood group $\mathrm{O}, \mathrm{A}$ and $B$ type. A positive reaction with $O$ cells indicates the presence of irregular Red cell antibodies (e.g. Anti-D, Anti-K, Anti-jka). If these are present, an identification with blood group $O$ cell panel is indicated.

Validation of the blood group of a blood donor requires two different techniques (at least one of which involves a BethVincent test and a Simonin test, two determinations made on two different samples, by two technicians, on two batches of different reagents [ $4 \mathrm{X} 2$ rule). In practice, this group is confirmed after two successive donations.

In many, if not most, SSA hospital blood banks pre-transfusion testing consists only of donor and recipient $\mathrm{ABO} / \mathrm{D}$ phenotyping, only with the Beth-Vincent method, and only once (not following the $4 \mathrm{X} 2$ rules). Cross-matching is done at the room-temperature saline method. Testing for irregular RBC alloantibodies is not performed, compatibility testing using an indirect antiglobulin technique or equivalent is rarely available, and most transfusion services do not have donors or unit's phenotype beyond the usual $\mathrm{ABO} / \mathrm{D}$. However, the limited number of studies that have examined the frequency of $\mathrm{RBC}$ alloimmunization in SSA demonstrate that approximately $6 \%$ of transfused patients develop clinically significant RBC alloantibodies. Rates like those reported in North American and European patients in general, or in sickle cell anaemia patients who receive partially phenotype blood [57].

It is therefore likely that blood that would be considered incompatible and therefore inappropriate for transfusion in HIC is frequently being transfused in LIC.

\section{PRODUCT ISSUANCE, AND TRANSPORT}

\section{Patient transfusion, monitoring, and follow-up}

Specific forms for requesting transfusions and issuing blood units are not consistently available or used throughout SSA, nor are written procedures for identifying patients, administering blood transfusions, or monitoring patients during transfusion. Challenges unknown in the HIC often exist, for example concerning patient identification, patients may not always use the same spelling for their names and often don't know their birth date, hospital identification bands are rarely used, and supplies of any sort for any step of the process, either on the hospital ward or in the blood bank, may run out. Finally, in the absence of patient monitoring during transfusion and/or active hemovigilance programs (see below, communications), it is likely that many acute transfusion reactions (ATR), including haemolytic reactions due to $\mathrm{ABO}$ incompatibility, go unrecognized and/or unreported. As an example (that is unlikely to be unique in SSA), in a prospective study of acute transfusion reactions that were performed in the major university hospital in Uganda, in a total of 500 transfusions, there were two ATR due to ABO compatibility errors and an additional mis-transfusion due to recipient identity error that was averted by study personnel [58].

\section{HEMOVIGILANCE PROGRAM}

Hemovigilance stands for the collective audit of transfusion safety, referring to both active and passive surveillance mechanisms to detect adverse outcomes among donors, transfusion, and recipients. This composite mechanism addresses all aspects of transfusion from recruitment and donation to post-transfusion surveillance in which sentinel events, including transfusion reactions and TTIs, are investigated.

Given limited resources, hemovigilance is a major challenge for transfusion services in SSA, where the tracking of patients, products, and outcomes remains deficient. 
Infectious risk is usually ascribed to blood donors, whereas the transmission hazard of component processing, like bacterial contamination, is often overlooked.

To be effective, uniformity of reporting, widespread adoption, and the means to extract data to guide regional policies and interventions are required. To this end, published data are scant, offering little evidence that nascent hemovigilance systems in SSA are functional.

Proficiency testing is also limited. Several external quality assurance studies have demonstrated the suboptimal performance of infectious testing at laboratories engaged in blood donor screening .

In conclusion Hemovigilance and QA are, consequently, central to blood safety.

\section{ACCREDITATION}

In an endeavour to improve this situation and enhance the quality and safety of blood transfusion in Africa, the African Society for Blood Transfusion (AfSBT), in collaboration with other international transfusion medicine organizations, in particular, the AABB, has recently developed the 'Stepwise Accreditation Programme' (SWAP). This system acknowledges the heterogeneous nature of SSA blood services and allows services of different levels to be considered for certification or accreditation, according to an expected standard for a given resource level. This also provides a structured path toward higher levels of accreditation.

\section{Education tool leading to accreditation}

A core activity for GAP is the evaluation of the National Society Blood Services (NSBS) through the Self-assessment (SA) questionnaire. This often highlights specific challenges experienced by NSBS in managing risk. As part of the SA feedback, GAP recommends that NSBS work towards blood service accreditation as a risk mitigation strategy.

Through this activity, it was identified that there is a need for suitable, internationally recognized minimum blood safety standards that fit in resource-limited settings, and for comprehensive blood service training programs linked to relevant accreditation standards.

This realization led to the birth of the GAP Modular Training Program - a comprehensive and cost-effective training program that strategically links to the requirements for NSBS.

Training customized to the requirements as outlined in the IFRC Blood Policy and the GAP Self- assessment establishes a strong foundation for NSBS to work towards achieving accreditation. In parallel, GAP was also undertaking a project assessing the AABB Fundamental Standards for Blood collection and Transfusion for their suitability in a resourcechallenged setting, which aligned perfectly to link with the modular training.

The development of the 'GAP Modular Training' program concept meets the following key objectives of GAP to develop and provide tools, guidelines and priority country assistance to National Society blood services most in need.

- Promote knowledge sharing, networking, and partnership among and between $\mathrm{RC} / \mathrm{RC}$ blood services and external partners, and.

- Work with the National Society (NS) to identify and manage risks concerning blood programs.

This program fits the recognized models of training and 'twinning' as an extremely effective strategy for transferring knowledge and expertise, which has been well established by GAP for many years, particularly in resource-limited settings where GAP has successfully applied this approach in its many previous country support projects.

Design: The Modular Training program has been designed to enable tailoring of specific activities and strategies through a series of multiple interchangeable modules, and, when delivered regionally, aims to connect NS blood services facing similar opportunities, challenges, and constraints. Five Core Areas are identified by the Global Advisory Panel (GAP): voluntary and no remunerated blood donation: (VNRBD), Infrastructure, Clinical, Quality, Governance, as depicted in Figure 3. These core areas are broken down into modules range from corporate governance and strategic management to fundamental operational systems, to transfusion practice, providing a complete vein to vein approach to training. And built from the detail (what, who, where, how, why). Additionally, it links to relevant national and international standards in all aspects of blood service delivery (such as $\mathrm{RC} / \mathrm{RC}$ policy, $\mathrm{AABB}$ fundamental standards and WHO guidelines), ultimately encouraging national societies to work towards achieving accreditation, or at least to operate within recognized minimum blood safety standards.

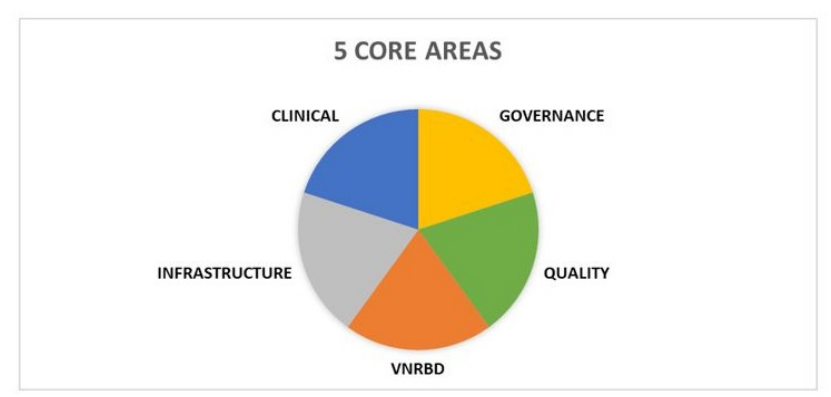

Figure 3: Five CORE AREAS within a blood service.

The program is coordinated and delivered by GAP using subject matter experts in the field of international blood programs, with additional region-specific technical expertise provided as required, to ensure relevance and suitability. To maximize accessibility to training, the mode of delivery has been considered, which can be used to deliver tailored modules in various formats to reach a wider or more remote audience.

\section{CONCLUSIONS}

To implement a small performing blood transfusion unit in an LMIC, the five Core Areas as identified by the Global Advisory Panel (GAP) must be met: voluntary and non-remunerated 
blood donation, an adapted Infrastructure, Clinically founded transfusions, Quality Assurance, and an efficient Governance. This not only requires sufficient resources, but education and accreditation as provided by the GAP are essential. What is usually forgotten, and can be a change maker, is the development of innovations in all stages of the blood transfusion chain and specifically focused on the local situation. This requires a global approach. There is a need for one or several worldwide centralized institutions, with extensive knowledge of tropical and subtropical environments, as well as limitations tied to socio-economically disadvantaged regions and where pre-qualification of tests and devices could be done. And wherever the entire transfusion chain can be simulated in a competence and training centre, focusing on the region where it will be applied.

\section{COMPLIANCE WITH ETHICAL STANDARDS}

In the realization of this review, there are no direct or indirect potential conflicts of interest to disclose. There are no sources of funding or acknowledgments. Also, research does not involve human or animal participants. Also, this review is dealing with the COPE guidelines.

\section{REFERENCES}

1. World Health Organization (2016) Global Status Report on Blood Safety and Availability.

2. Barro L, Drew VJ, Poda GG, Tagny CT, El-Ekiaby M, Owusu-Ofori $\mathrm{S}$, et al. Blood transfusion in sub-Saharan Africa: understanding the missing gap and responding to present and future challenges. Vox Sang. 2018;113:726-736.

3. Bates I, Hassall O. Should we neglect or nurture replacement blood donors in sub-Saharan Africa? Biologicals. 2010;38:65-67.

4. Bates I, Hassall O, Mapako T. Transfusion research priorities for blood services in sub-Saharan Africa. $\mathrm{Br} \mathrm{J}$ Haematol. 2017; 177:855-863.

5. Rukundo HAM, Taremwa IM, Muwanguzi E, Ndyomubantu M, Byarugaba F, Natukunda B. Assessment of the quality of stored blood for transfusion at mbarara regional referral hospital, Southwestern Uganda. J Blood Med. 2019;10:161-169.

6. Flanagan P. Blood Donation - Incentives and Inducements: where to draw the line? ISBT Sci Ser. 2020;15:19-22.

7. Atinuke Fasola F, Fadimu PA, Akpan VO. A Seven Year Review of the Seroprevalence of Transfusion Transmitted Infections in a Hospital Based Blood Bank in Ibadan, Nigeria. Clin Med Res. 2017;6:1.

8. Allain JP, Sibinga CTS. Family donors are critical and legitimate in developing countries. Asian J Transfus Sci. 2016;10:5-11.

9. Bloch EM, Vermeulen M, Murphy E. Blood Transfusion Safety in Africa. 2013;26:164-180.

10. Asenso-Mensah K, Achina G, Appiah R, Owusu-Ofori S, Allain JP. Can family or replacement blood donors become regular volunteer donors? Transfusion. 2014;54:797-804.

11. Allain JP. Current approaches to increase blood donations in resource-limited countries. Transfus Med. 2019;29:297-310.

12. Dujardin B, Kegels G, Buvé A, Mercenier P. Tuberculosis control: did the programme fail or did we fail the programme? Trop Med Int Health. 1997;2:715-718.

13. Mounier-Jack S, Griffiths UK, Closser S, Burchett H, Marchal B. Measuring the health systems impact of disease control programmes: a critical reflection on the WHO building blocks framework. 2011.

14. Shirley Owusu-Ofori M. Letters to the Editor. Transfusion. 2005;5:133-140.

15. Allain JP, Sarkodie F, Boateng P, Asenso K, Kyeremateng E, Owusu-Ofori S. A pool of repeat blood donors can be generated with little expense to the blood center in sub-Saharan Africa. Transfusion. 2008 48:735-741.

16. Kenyon CR, Delva W. It 's the network, stupid]: a population 's sexual network connectivity determines its STI prevalence. 2019.

17. Tagny CT, Nguefack-Tsague G, Fopa D, Ashu C, Tante E, Ngo Balogog P, et al. Risk factors for human immunodeficiency virus among blood donors in Cameroon: evidence for the design of an Africa-specific donor history questionnaire. Transfusion. 2017;57:1912-1921.

18. El Ekiaby M, Lelie N, Allain JP. Nucleic acid testing [NAT] in high prevalence-low resource settings. Biologicals. 2010;38:59-64.

19. Mbanya D. Use of quality rapid diagnostic testing for safe blood transfusion in resource-limited settings. Clin Microbiol Infect. 2013;19:416-421.

20. Prugger C, Laperche S, Murphy EL, Bloch EM, Kaidarova Z, Tafflet M, et al. creening for transfusion transmissible infections using rapid diagnostic tests in Africa: A potential hazard to blood safety? Vox Sang. 2016;110:196-198.

21. Bloch EM, Shah A, Kaidarova Z, Laperche S, Lefrere JJ, van Hasselt J, et al. A pilot external quality assurance study of transfusion screening for HIV, HCV and HBsAG in 12 African countries. Vox Sang. 2014;107:333-342.

22. Abdelrazik AM, Ezzat Ahmed GM. Priority needs and wisdom strategy for blood transfusion safety in developing low-resource countries. Transfus Apher Sci. 2016;54:147-149.

23. Hogman C, Gong J, Eriksson L, Hambraeus A, Johansson C. White cells protect donor blood against bacterial contamination. Transfusion. 1991;31:620-626.

24. Adegoke AO, Akanni OE. Survival of treponema pallidum in banked blood for prevention of syphilis transmission. N Am J Med Sci. 2011;3:329-332.

25. Nadelman R, Sherer C, Mack L, Pavia C, Wormser G. Survival of Borrelia burgdorferi in human blood stored under blood banking conditions. Transfusion. 1990;30:298-301.

26. Ruiz J, Silva W, Pons MJ, Del Valle LJ, Tinco CR, Casabona VD, et al. Long time survival of Bartonella bacilliformis in blood stored at $4{ }^{\circ} \mathrm{C}$. A risk for blood transfusions. Blood Transfus. 2012;10:563-564.

27. Martin DL, Goodhew B, Czaicki N, Foster K, Rajbhandary S, Hunter S, et al. Trypanosoma cruzi survival following cold storage: Possible implications for tissue banking. PLoS One 9. 2014.

28. Cursino-Santos JR, Alhassan A, Singh M, Lobo CA. Babesia: Impact of cold storage on the survival and the viability of parasites in blood bags. Transfusion. 2014;54:585-591.

29. Césaire R, Kérob-bauchet B, Bourdonné O, Maier H, Amar KO. Components in a Routine Setting. 2004:44:42-48.

30. Mettille FC, Salata KF, Belanger KJ, Casleton BG, Kelly DJ. Reducing the risk of transfusion-transmitted rickettsial diseaseby WBC filtration, using Orientia tsutsugamushiin a model system. Transfusion. 2000;40:290-296.

31. Proctor MC, Leiby DA. Do leukoreduction filters passively reduce the transmission risk of human granulocytic anaplasmosis? Transfusion. 2015;55:1242-1248.

32. Dzik W. Use of leukodepletion filters for the removal of bacteria. Immunol Invest. 2019;24:95-115.

33. Zavizion B, Pereira M, de Melo Jorge M, Serebryanik D, Mather $\mathrm{TN}$, Chapman J, et al. Inactivation of protozoan parasites in red 
blood cells using INACTINE PEN110 chemistry. Transfusion. 2004;44:731-738.

34. Schuetz AN, Hillyer KL, Roback JD, Hillyer CD. Leukoreduction filtration of blood with sickle cell trait. Transfus Med Rev. 2004;18:168-176

35. Economopoulou A, Dominguez M, Helynck B, Sissoko D, Wichmann O, Quenel P, et al. Atypical Chikungunya virus infections: clinical manifestations, mortality and risk factors for severe disease during the 2005-2006 outbreak on Réunion. Epidemiol Infect. 2009;137:534-541.

36. Allain JP, Owusu-Ofori AK, Assennato SM, Marschner S, Goodrich RP, Owusu-Ofori S. Effect of Plasmodium inactivation in whole blood on the incidence of blood transfusion-transmitted malaria in endemic regions: The African Investigation of the Mirasol System [AIMS] randomised controlled trial. Lancet. 2016;387:1753-1761.

37. Tonnetti L, Thorp AM, Reddy HL, Keil SD, Goodrich RP, Leiby DA. Evaluating pathogen reduction of Trypanosoma cruzi with riboflavin and ultraviolet light for whole blood. Transfusion. 2019;52:409-416.

38. Cap AP, Pidcoke HF, Keil SD, Staples HM, Anantpadma M, Carrion $\mathrm{R}$, et al. Treatment of blood with a pathogen reduction technology using ultraviolet light and riboflavin inactivates Ebola virus in vitro. Transfusion. 2016;56:S6-S15.

39. Jimenez-Marco T, Cancino-Faure B, Girona-Llobera E, Alcover MM, Riera C, Fisa R. The effectiveness of riboflavin and ultraviolet light pathogen reduction technology in eliminating Trypanosoma cruzi from leukoreduced whole blood. Transfusion. 2017;57:1440-1447.

40. Allain JP, Goodrich R. Pathogen reduction of whole blood: utility and feasibility. Transfus Med. 2017;27:320-326.

41. Qadri SM, Chen D, Schubert P, Perruzza DL, Bhakta V, Devine DV, et al. Pathogen inactivation by riboflavin and ultraviolet light illumination accelerates the red blood cell storage lesion and promotes eryptosis. Transfusion. 2017;57:661-673.

42. Owusu - Ofori AK, Parry C, Bates I. Transfusion Transmitted Malaria in Countries Where Malaria Is Endemic: A Review of the Literature from Sub - Saharan Africa. Clin Infect Dis. 2010;51:1192-1198.

43. Tapko J-B, Mainuka P, Diarra-Nama AJ. Status of blood safety in the WHO African Region: report of the 2006 survey. Africa [Lond]. 2009;1-50.

44. Tagny CT, Diarra A, Yahaya R, Hakizimana M, Nguessan A, Mbensa $G$, et al. Characteristics of blood donors and donated blood in sub-Saharan Francophone Africa. Transfusion. 2009;49:1592-1599.

45. Garrigue D, Godier A, Glacet A, Labreuche J, Kipnis E, Paris C. French lyophilized plasma versus fresh frozen plasma for the initial management of trauma-induced coagulopathy: a randomized openlabel trial. J Thromb Haemost. 2018;16:481-489.

46. Babigumira JB, Lubinga SJ, Castro E, Custer B. Cost-utility and budget impact of methylene blue-treated plasma compared to quarantine plasma. Blood Transfus. 2018;16:154-162.

47. Walsh M, Fries D, Moore E, Moore H, Thomas S, Kwaan HC. Whole Blood for Civilian Urban Trauma Resuscitation: Historical, Present, and Future Considerations. Semin Thromb Hemost. 2020;46:221-234.

48. Ala F, Allain JP, Bates I, Boukef K, Boulton F, Brandful J. External Financial Aid to Blood Transfusion Services in Sub-Saharan Africa: A Need for Reflection. PLoS Med. 2012;9:e1001309.

49. Sood R, Raykar N, Till B, Shah H, Roy N. Walking blood banks: an immediate solution to rural India's blood drought. Indian J Med Ethics. 2017;III:134-137.

50. Nébié K, Ouattara S, Sanou M, Kientega Y, Dahourou H, Ky L. Poor procedures and quality control among nonaffiliated blood centers in Burkina Faso: An argument for expanding the reach of the national blood transfusion center. Transfusion. 2011; 51:1613-1618.

51. Opare-Sem O, Bedu-Addo G, Karikari P, Boateng P, Sarkodie F, Rahman R. Fourteen-year experience of a tertiary hospital transfusion committee in West Africa. Transfusion. 2014;54:2852-2862.

52. Kabatereine NB, Malecela M, Lado M, Zaramba S, Amiel O, Kolaczinski JH. How to [or not to] integrate vertical programmes for the control of major neglected tropical diseases in Sub-Saharan Africa. PLoS Negl Trop Dis. 2010;4:1-8.

53. The Clinical Use of Blood in General Medicine Obstetrics Paediatrics Surgery \&amp; Anaesthesia Trauma \&amp; Burns.

54. Pauker SG, Kassirer JP. THE THRESHOLD APPROACH TO CLINICAL DECISION MAKING. N Engl J Med. 1980;302:1109-1117.

55. Natukunda B. Red blood cell alloimmunization and antigen matching in sickle cell disease - the African perspective. ISBT Sci Ser. 2012;7:129-133.

56. Waiswa MK, Moses A, Seremba E, Ddungu H, Hume HA. Acute transfusion reactions at a national referral hospital in Uganda: A prospective study. Transfusion. 2014;54:2804-2810.

57. Hassall O, Maitland K, Pole L, Mwarumba S, Denje D, Wambua K. Bacterial contamination of pediatric whole blood transfusions in a Kenyan hospital. Transfusion. 2009;49:2594-2598.

58. Laperche S. Multinational assessment of blood-borne virus testing and transfusion safety on the African continent. Transfusion. 2013;53:816-826. 